\title{
Revisiting Brain Tuberous Sclerosis Complex in Rat and Human: Shared Molecular and Cellular Pathology Leads to Distinct Neurophysiological and Behavioral Phenotypes
}

\author{
Viera Kútna $^{1}$ • Valerie B. O'Leary ${ }^{2}$ Ehren Newman ${ }^{3}$ - Cyril Hoschl ${ }^{1,4} \cdot$ Saak V. Ovsepian ${ }^{1,4}$ (DD
}

Accepted: 21 December 2020 / Published online: 4 January 2021

(C) The American Society for Experimental NeuroTherapeutics, Inc. 2021

\begin{abstract}
Tuberous sclerosis complex (TSC) is a dominant autosomal genetic disorder caused by loss-of-function mutations in TSC1 and TSC2, which lead to constitutive activation of the mammalian target of rapamycin C1 (mTORC1) with its decoupling from regulatory inputs. Because mTORC1 integrates an array of molecular signals controlling protein synthesis and energy metabolism, its unrestrained activation inflates cell growth and division, resulting in the development of benign tumors in the brain and other organs. In humans, brain malformations typically manifest through a range of neuropsychiatric symptoms, among which mental retardation, intellectual disabilities with signs of autism, and refractory seizures, which are the most prominent. TSC in the rat brain presents the first-rate approximation of cellular and molecular pathology of the human brain, showing many instructive characteristics. Nevertheless, the developmental profile and distribution of lesions in the rat brain, with neurophysiological and behavioral manifestation, deviate considerably from humans, raising numerous research and translational questions. In this study, we revisit brain TSC in human and Eker rats to relate their histopathological, electrophysiological, and neurobehavioral characteristics. We discuss shared and distinct aspects of the pathology and consider factors contributing to phenotypic discrepancies. Given the shared genetic cause and molecular pathology, phenotypic deviations suggest an incomplete understanding of the disease. Narrowing the knowledge gap in the future should not only improve the characterization of the TSC rat model but also explain considerable variability in the clinical manifestation of the disease in humans.
\end{abstract}

Key Words mTOR signaling $\cdot$ autism spectrum disorders $\cdot$ hamartoma $\cdot$ neoplasia $\cdot$ TSC1 $\cdot$ TSC $2 \cdot$ refractory epilepsy

\section{Introduction}

Tuberous sclerosis complex (TSC) is a rare autosomal dominant genetic disease. It manifests through multisystem

Viera Kútna

viera.kutna@nudz.cz

Saak V. Ovsepian

saak.ovsepian@nudz.cz

1 Department of Experimental Neurobiology, National Institute of Mental Health, Topolová 748, 25067 Klecany, Czech Republic

2 Department of Medical Genetics, Third Faculty of Medicine of Charles University, Ruská 87, 10000 Prague, Czech Republic

3 Department of Psychological and Brain Sciences, Indiana University, Bloomington, IN, USA

4 Department of Psychiatry and Medical Psychology, Third Faculty of Medicine of Charles University, Ruská 87, 100

00 Prague, Czech Republic pathology associated with the development of noncancerous tumors in the brain, kidneys, heart, liver, eyes, lung, and skin. Neurological and psychiatric symptoms such as anxiety, depressed mood, attentional and intellectual disabilities, communication problems, and refractory seizures are among the most recognized manifestations of the disease, along with developmental retardation and abnormalities of lung, skin, and kidney functions. TSC malformations in the brain, which arise due to uncontrolled tissue growth, result from hyperactivity of mammalian target of rapamycin $\mathrm{C} 1$ (mTORC1) complex in neurons, glial cells, and ventricular ependyma [1]. Currently, TSC is classified within mTOR-related disorders, or so-called "mTORopathies," caused by mutations in regulatory genes in the mTOR pathway, e.g., TSC1, TSC2, AKT3, DEPDC5, and $S T R A D A$ [2-4]. The condition was originally described in humans as a multisystem pathology by Bourneville in the 1880s. Cortical tubers known also as hamartia are the most frequently encountered substrate for seizures, which are prevalent in $90 \%$ of patients. Histopathological features of brain 
tubers include focal dysplasia with irregular cellular organization and loss of lamination, and presence of enlarged neuronlike structures containing glassy eosinophilic cytoplasm and euchromatic single or multiple nuclei, as well as enlarged reactive astrocytes occurring mainly in the cortex and underlying white matter $[5,6]$.

The discovery of hereditary renal adenomas and adenocarcinomas in Eker rats carrying a spontaneous germline loss-offunction mutation in $t s c 2\left(t s c 2^{+/}\right)$facilitated preclinical studies of the histopathological and molecular alterations related to TSC $[7,8]$. The formation of multiple kidney tumors is the most characteristic feature for this model, with lesions in the brain, spleen, and uterus also described [9]. Brain malformations in $t s c 2^{+/-}$rats, like in humans, have agedependent onset and develop in 30 to $65 \%$ of cases $[10,11]$. Similar to humans, in Eker rats, TSC lesions are associated with hyperactivity of mTORC1 signaling resulting from spontaneous loss-of-function germline $t s c 2$ mutations. As the homozygous $\left(t s c 2^{-/}\right.$) variant is lethal on $\sim 13$ days of embryogenesis [12], all Eker rats used as research models are heterozygotes, carrying one nonfunctional allele. Importantly, the onset of neoplastic changes in the Eker model can be influenced by environmental factors, a finding that prompted the two-hit hypothesis of TSC, which assumes at least two causatives $[13,14]$. The first is genetic aberrations, either inherited (predisposing) or occurring spontaneously in somatic cells. The second hit is caused by environmental exposure (e.g., ionizing radiation, chemical carcinogens), which increases the risk of the disease by altering the proliferation and signaling mechanisms in cells affected by the mutations, as well as through suppression of immune response, or other alterations favoring the onset of the pathology.

The discrete cellular and molecular impairments with the well-defined role of environmental and genetic factors, and robust neurological phenotype render the Eker rats an excellent model for the analysis of the biology of human TSC malformations, and potentially also other benign brain tumors, with therapeutic intervention. In light of recent advances in tissue grafting methods and the arrival of new genetic models of brain neoplasia, it is important to note that along with numerous methodical and translational advantages, these emerging models have also considerable limitations [15-17]. Indeed, grafting brain tumors for research involves a highly invasive procedure, complex genetic and immunological incompatibility issues, with failure to replicate the insidious onset and gradual progression of molecular and cellular pathology [18-21]. In this context, the spontaneous brain tumor models such as Eker rats have much to offer, in replicating the molecular pathobiology, the initiation and development of the disease without immunological and genetic compatibility issues. Throughout this article, we revisit human and rat TSC and relate their histopathological, neurobehavioral, and electrophysiological phenotypes. We highlight several novel facets of $t s c 2^{+/-}$rats, which offer a range of attractive clinical and translational applications for neuro-oncology and clinical neurology and discuss the limitations of the model and areas for future advancements.

\section{Molecular Biology and Pathobiology of TSC}

TSC1 and TSC2 have been identified using positional cloning and localized to chromosomal regions $9 \mathrm{q} 34$ and $16 \mathrm{p} 13.3$, respectively [22-24]. Products of these genes, hamartin (TSC1) and tuberin (TSC2), interact to control the activity of Ras homolog Rheb small GTPase, which plays a key role in regulating mTORC1, a kinase that controls protein synthesis, cell growth and proliferation, cell cycle, and energy metabolism [25, 26] (Fig. 1a-c). Hamartin is known to stabilize tuberin and prevent its degradation, while tuberin holds the functional GAP signaling domain and serves as a GTPase-activating protein (GAP) for Rheb, making TSC1 and TSC2 complex obligatory for the regulation of the TSC pathway [28]. Both proteins are necessary for physiological mTORC1 functioning; therefore, a loss of function of either of these is sufficient to cause pathobiological changes and clinical phenotype of the TSC.

TSC1 consists of 23 exons, of which 21 are coding, with exons 1 and 2 also undergoing alternative splicing. Its product hamartin consists of 1164 amino acids $(130 \mathrm{kDa})$ and has no homology with tuberin or any other vertebrate protein $[23$, 29]. In addition to binding and stabilizing tuberin, hamartin also interacts with the N-terminal of the ezrin-radixin-moesin (ERM) family of highly regulated actin-binding proteins and plays a key role in cell adhesion, mobility, and activation of the small GTP-binding protein Rho [30-32]. ERM proteins form cross-links between actin filaments and the plasma membrane, playing a key role in organizing protein-lipid rafts in the surface membrane, linking transmembrane proteins and cytoskeleton scaffolds [33]. The interaction of hamartin with moesin, a protein concentrated in the neuronal growth cone, suggests a key role for the hamartin-tuberin complex in the axonal sprouting and motility, and in neurite elongation [34]. Finally, hamartin is known to interact with intermediate neurofilament L [31], with stabilizing effects on the cytoskeleton.

Unlike TSC1, TSC2 has 41 exons, with 25, 26, and 31 exons subject to alternative splicing [35]. It translates into tuberin, which is an 1807 amino acid protein with a molecular mass of $198 \mathrm{kDa}$. Residues encoded by exons 34 to 38 have significant homology to the human catalytic domain of Rap1 GAP and murine Spa1 GAPs [36]. Rap1 has oncogenic and mitogenic properties, and plays a potential role in mitogenactivated protein kinase (MAPK)-mediated neuronal differentiation, and downregulates the activity of Ras-related proteins Rap1a and Rap1b [37-39]. Importantly, tuberin also contains a homology region to the GAP3 [40], and as such 
a

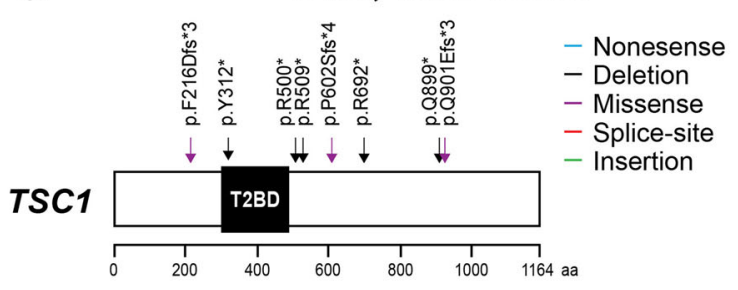

C

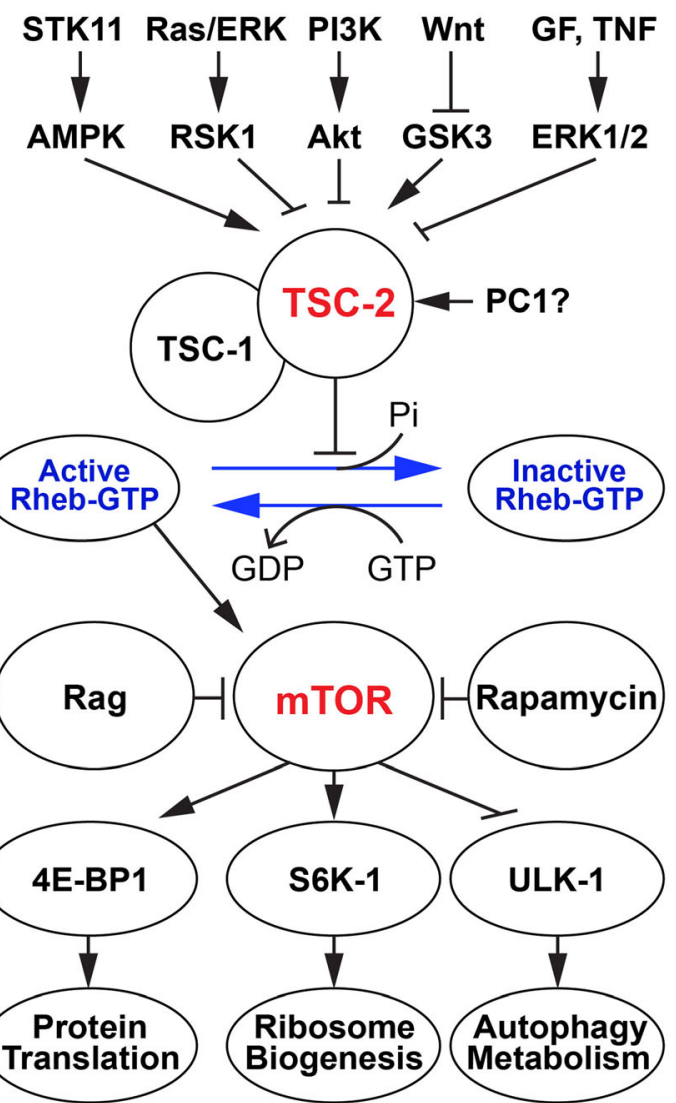

Fig. 1 Map of TSC1 and TSC2 point (p.) mutations identified in subependymal giant cell astrocytoma (SEGA) tumors. Arrow colors present different mutation types, as indicated. (a) Maps of TSC1 (upper) and TSC2 (lower) mutations, respectively. Two TSC2 mutations differ by a single nucleotide position in the same amino acid (p.R611Q/p.R611W). Reused with permission from Bongaarts et al. [27]. (b) Schematic of the TSC1 (upper; hamartin) and TSC2 (lower; tuberin) proteins. The functional domains on TSC1 and TSC2 are depicted with numbers representing amino acid residues on the full-length human proteins.

is a potent cell cycle regulator, controlling protein translation at the G1 to S phase [41, 42]. Studies of tuberin distribution have shown its enrichment in the nucleus and cytoplasm [43], as well as in the Golgi apparatus [44]. Of note, maintenance of TSC2 cytoplasmic location depends on the level of supply of amino acids and nerve growth factors, with one of two in short supply triggering the sorting of TSC1/2 to the lysosomes [45, 46].

As noted, hamartin and tuberin are coexpressed in cells of several organs, including the brain; therefore, loss-of-function mutations in TSC1 and TSC2 can affect a wide range of cellular processes $[47,48]$. Several major signaling pathways, including PI3K-Akt, ERK, and AMPK, are interlinked and regulate the activity of the hamartin/tuberin complex, which can be stimulated by multiple growth factors (e.g., TNF $\alpha$, IGF, or BDNF). Activated PI3K leads to the recruitment of PDK1 and the serine/threonine protein kinase Akt, and stimulates the phosphorylation/activation of Akt by PDK1.
T2BD = TSC2-binding domain; Coil = predicted coiled-coil domain; T1BD = TSC1-binding domain; GAP = GAP domain homologous with that in Rap1-GAP. (c) Overview of the key receptors and molecular mechanisms involved in signaling pathways that converge at the TSC1-2 complex, which via regulating Rheb-GTP state controls the mTOR activity. In turn, mTOR regulates 4E-BP1, S6K-1, and ULK-1 activity, controlling protein translation, ribosome biogenesis, and autophagy, and contributing to the histopathological, functional, and neurobehavioral phenotypes of TSC

Stimulation of Akt, in turn, downregulates hamartin by phosphorylating tuberin at five consensus sites [49-52]. A second major kinase, which also can phosphorylate and inhibit tuberin, is the extracellular signaling-regulated kinase (ERK) [53]. ERK phosphorylation of tuberin appears to play an important role in EphA receptor-mediated regulation of TSC functions [54]. Both Akt and ERK levels are found to be increased in cortical and subcortical tubers, while inhibition of tuberin by these kinases has been proposed to present a post-translational mechanism that may further amplify the loss of function of TSC2 [53, 55]. Tuberin can also be phosphorylated by AMPK but at a different set of residues than that of Akt and ERK, and can increase the ability of the TSC complex to inhibit mTORC1 activity, protecting cells from excessive energy usage during low energy states [56, 57]. Importantly, hamartin is also negatively regulated by IKK- $\beta$, which physically interacts with and phosphorylates it at Ser487 and Ser511 residues in response to activation of inflammatory 
pathways [58]. As the PI3K/PTEN mTORC1 signaling plays an important role in controlling cell division by growth factors, loss of function of hamartin and tuberin lead to out-ofcontrol activation of the mTORC1 pathway, due to increasing Rheb-GTP levels, causing cell size increase and tissue growth [59] (Fig. 1c). The latter involves key effectors of mTORC1 such as eukaryotic initiation factor 4E-binding protein (4E$\mathrm{BP} 1$ ) and the $70-\mathrm{kD}$ ribosomal protein $\mathrm{S} 6$ kinase (S6K1), which cooperate to coordinate the behavior of both eukaryotic initiation factors and the ribosome, regulating protein synthesis. In agreement with such mechanisms, in TSC hamartomas, there is a significant increase in phospho-S6 (pS6) activity $[60,61]$, which is a key component of the $40 \mathrm{~S}$ ribosomal submit, and is therefore involved in protein translation.

The majority of loss-of-function TSC mutations that cause symptoms of TSC occur spontaneously in either TSC1 or TSC2, with mutations in TSC2 in TSC patients about five times more common than in TSC1. These mutations are typically represented by a mix of nonsense, deletion, missense, or insertions, involving nearly all of the exons [62, 63] (Fig. 1a, b). Because hamartin and tuberin cooperate to form a functional complex, the higher rates of clinical cases with TSC 2 mutations suggest a greater exposure of the TSC2 gene to second "hits," which increases the likelihood of the disease onset.

\section{Human TSC Brain Pathology}

The prevalence of TSC in humans is estimated at $1 / 6000$, with approximately two-thirds of all reported cases having no family history [64]. The disease has an early onset, with TSC loss of function manifested via a range of neurological and psychiatric symptoms. Due to complex and common comorbidities, however, neuropsychiatric symptoms of TSC are occasionally underrated and misdiagnosed [65], or overlooked until advanced disease stages, manifested in intellectual retardation during adolescence and early adulthood [66]. Behavioral impairments such as difficulties with sleep, hyperactivity, and impulsive conduct related to mood swings, anxiety, and occasional bursts of aggression are prevalent in the majority of TSC cases [67]. TSC malfunctions seem to also be associated with signs of autism spectrum disorder, which has been recognized in up to $60 \%$ of affected subjects and more commonly linked with TSC2 loss of function [68]. Finally, cases of schizophrenia and depression are documented more frequently in TSC subjects as compared to the general population [67, 69]. The most widespread neuropsychiatric sign of TSC, nonetheless, is epilepsy, which is typically resistant to drugs and is related to advanced cortical tubers [70, 71].

Based on the location, brain TSC lesions are classified into three groups: 1) cortical and subcortical tubers, 2) subependymal nodules (SENs), and 3) giant cell astrocytoma
(SEGA) [72, 73]. All these are benign malformations and share histopathological features. Importantly, all three brain TSC classes also display characteristic ventricle extension towards lesions, suggesting that the ventricular ependyma represents the site of disease initiation with abnormal cell proliferation and migration in the course of development. Consistent with this view, radial white hyperintense matter tracts are occasionally visible in structural MRI scans of TSC brains, along with heterotrophic gray matter [74]. The majority of cortical tubers appear as well-confined lesions characterized by dysplasia, loss of myelinated fibers, and increased concentration of glial cells and processes [5,75], with the presence of corpora amylacea in the molecular layer [76]. Cortical tubers occur typically in the cerebrum (i.e., are supratentorial), unilaterally or bilaterally, and are most frequent in frontal and parietal lobes [72, 73, 77]. Figure 2a, b shows typical MRI images of a tuber with a large dysplastic zone in the parieto-occipital region of a 5-month-old girl, with representative EEG recordings of reoccurring epileptic seizures captured with scalp EEG (Fig. 2c). Further characteristic examples of MRI-PET showcasing cortical and subcortical tubers in both temporal lobes with related metabolism changes in the brain of a 10-month-old boy and intractable seizures are presented in Fig. 2d. Figure 2e, on the other hand, shows rare forms of cerebellar tubers on T2-weighted MRI from a 17year-old boy, with superimposed images highlighting an area of hypometabolism matching that of the tuber region of a 3year-old girl with seizures [79]. Note multiple areas of hypometabolism corresponding to tubers, suggesting that they might be epileptogenic or contain necrotic changes. Histopathologically, cortical tubers comprise a mixture of abnormally large cells with neuronal and glial phenotype (known also as balloon cells) and characteristics of focal dysplasia $[5,76]$. Despite their disproportionately large size, the giant neuron-like cells display features of regular neurons, i.e., prominent Nissl substance, round and centrally localized nucleus, and distinct nucleolus as well as microtubule-associated protein 2 (MAP-2) and MAP-1B immune-positivity. Orphan giant cells lacking Nissl substance and eccentric nucleolus, and nonreactive to GFAP, vimentin, nestin, and MAP2 represent the second most distinguishing feature of lesions $[75,77$, 80]. Immunohistochemical staining shows occasional reactivity of these cells for neurofilaments (phosphorylated and nonphosphorylated), calbindin D-28K, and synaptophysin. Some giant cells can appear also weakly GFAP positive and contain adhesion molecule CD44 and microglial marker CD68 [77]. In contrast with normal size cells in the cortex, these giant cells can be enriched with hamartin and tuberin [76, 81].

SENs, on the other hand, are primarily comprised of atypical and swollen multinucleated cells of a glial phenotype [82]. In most subependymal malformations, cells with glial features develop small and distinct protrusions into the ventricular 


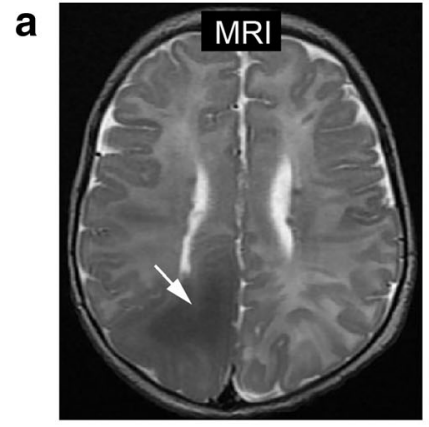

b

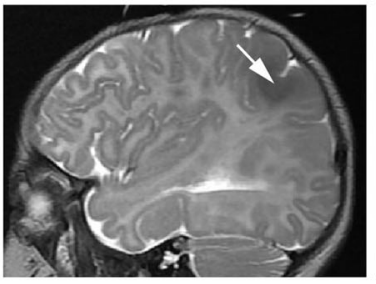

c

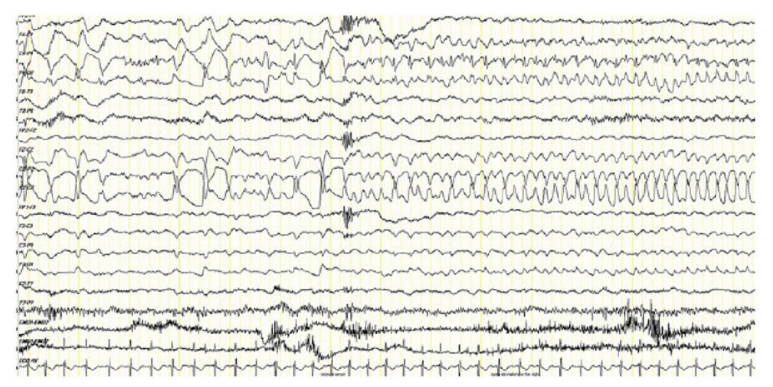

d
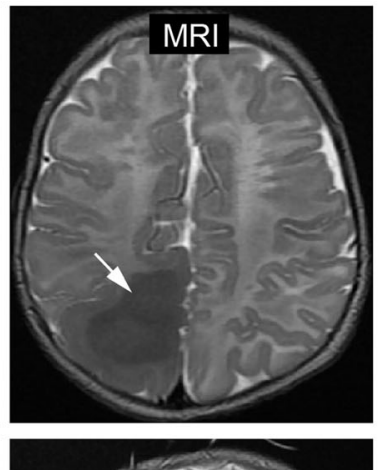
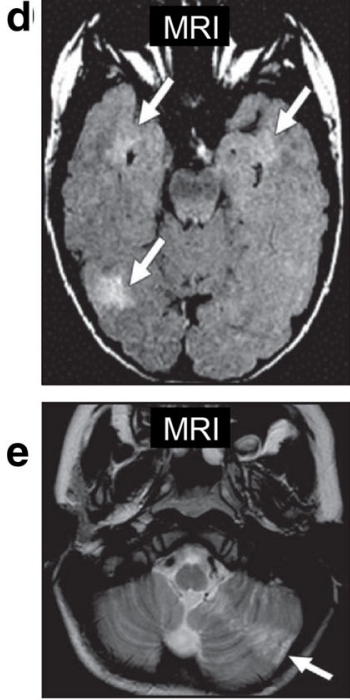

f

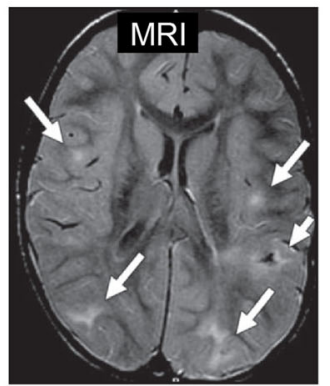

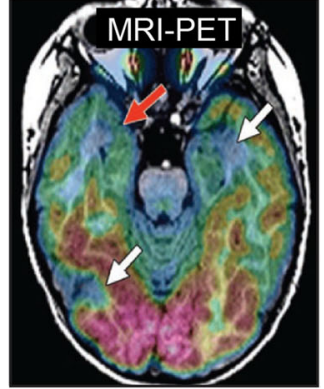
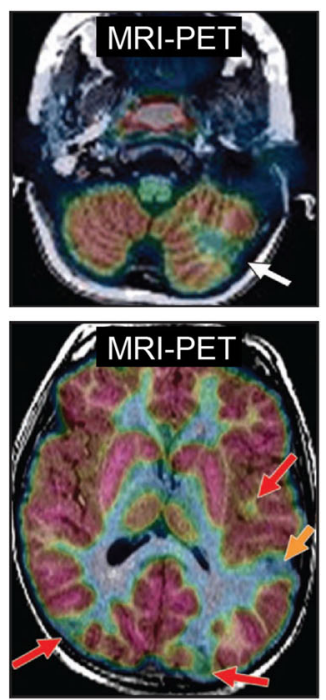

Fig. 2 Cross-section (a) and transverse (b) MRI images showing a large dysplastic zone (left upper-lower, arrow) in the right parieto-occipital brain region of a female patient with TSC2 mutation. By 5 months of age, this patient presented motor asymmetry with left side impairment and frequent spasms despite drug treatment. At the age of 7 months, this patient underwent an invasive treatment leading to a parieto-occipital disconnection (right upper-lower, arrow). Subsequently, the patient was seizure free for almost 6 years under treatment. (c) Representative recording traces of an EEG at the onset of a seizure in the same patient with TSC2 mutation as described above. Reproduced with modification from Fohlen et al. [78]. (d) Axial FLAIR MR image showing cortical and subcortical tubers (left, arrows) in both temporal lobes of a 10-month-old boy with tuberous sclerosis complex and intractable seizures. Axial MRI/ PET fusion image showing multiple areas of hypometabolism

cavity, with parts of the lesion occasionally becoming calcified with aging and stop growing, while others grow markedly in size [83]. Such enlargement occasionally signals the transition of small subependymal nodules into giant cell astrocytomas. The latter, if localized close to ventricular channels, carry a risk of obstructive hydrocephalus and death if untreated. Histopathologically, the difference between SEN and SEGA is rather indiscernible $[83,84]$, with their anatomical location usually used as the main criteria of distinguishment. Under these criteria, SENs typically localize in the periventricular region [85], near to the caudate nucleus and caudothalamic sulcus $[5,72,73]$. Also, SENs frequently are enriched with GFAP-positive spindle cells and contain small groups of vimentin immunoreactive balloon cell-like structures, although results with GFAP and neuronal markers corresponding to tubers (right, white arrows). Tuber (red arrow) in the right anterior temporal lobe shows a disproportionately large area of hypometabolism, indicating that the zone is probably epileptogenic. (e) The axial T2-weighted image shows left cerebellar tuber with a typical wedge-shaped configuration (left, arrow). Axial MRI/PET fusion image showing area of hypometabolism approximately equal in size to the cerebellar tuber (right, arrow). (f) Axial FLAIR MR image showing multiple bilateral cortical and subcortical tubers (left, arrows). Axial PET/MRI fusion image showing multiple areas of hypometabolism (red arrows) corresponding to tubers. Tuber in the left posterior temporal lobe shows disproportionate area hypometabolism (orange arrow) indicating that the zone is probably epileptogenic. Images were taken from a 17year-old boy with TSC and intractable seizures. Reproduced with permission from Kalantari and Salamon [79]

synaptophysin, MAP2, and neurofilament staining, considerably varying from study to study $[77,85]$.

Symptomatic SEGA, while occurring in $\sim 10 \%$ of TSC patients, is considered as the main cause of mortality and shortened lifespan [83]. Histochemical analysis has shown that SEGA contains three main cell types: spindle cells, middlesized ganglion-like epithelioid cells [86], and giant cells [87]. Computed tomography scans typically reveal numerous cases of asymptomatic SEGA in the brain of TSC patients, often occurring bilaterally [84]. Depending on the location, SEGA can interfere with CSF circulation and lead to hydrocephalus and death [88]. Of note, in the majority of cases, SEGAs are confined in close vicinity to the caudate nucleus near the foramen of Monro or lateral ventricles [72, 76, 83, 85, 86, 89]. Like in SENs, the majority of SEGA cells display 
morphologic features of giant glial cells, with an expression of GFAP and S-100 protein $[77,86,87,90]$. These atypical and unusually large cells express brain lipid-binding protein (BLBP) [91, 92], which plays a key role during the development of radial glia and migration of immature neurons [93]. Remarkably, a considerable number of SEGA cells also show positivity for an array of neuronal markers, i.e., SMI33, TpNF1A3, TUJ1, CL-300 [87], neuron-specific enolase (NSE), synaptophysin [77, 86], and doublecortin [91], as well as markers for somatostatin, metenkephalin, 5-HT, $\beta$-endorphin, and neuropeptide $\mathrm{Y}$ [87]. Of note, giant cells of SEGAs, but not spindle and epithelioid cells, express tuberin [81], whereas subependymal cells show a significant reduction in the hamartin expression [94].

As emerges from the studies reviewed above, TSC in the human brain reveals a highly complex histochemical and molecular profile, which together with specific anatomical locations determine the neurocognitive and behavioral outcome. While replication of specific features of benign TSC-like neoplasms in animal models using xenografts might be feasible, reproducing neuropathological and behavioral phenotypes of TSC in their entirety would be a major challenge. The autochthonous TSC malformations of Eker rats, therefore, present a useful research model for enabling the analysis and characterization of a range of neuropathological phenotypes, influenced by genetic and environmental factors.

\section{Brain Malformations in the TSC Eker Rat: an Overview}

The Eker rat model of human TSC is characterized by a spontaneous loss-of-function tsc 2 mutation that causes inactivation of one of the alleles, due to intronic transposition of an intracisternal-A particle into the $t s c 2$ locus. This results in the production of a nonfunctional (truncated) tuberin that leads to an out-of-control mTORC1 activation. Over decades of $t s c 2^{+/-}$research in Eker rats, neurologic deficits reported were minimal, with until recently, only a single study showing the presence of brain tubers [95]. The absence of brain lesions in the majority of $t s c 2^{+--}$rats invoked the two-hit hypothesis, whereby an initial germline loss-of-function mutation is compounded by a somatic mutation, which precipitates the onset of the disease. This hypothesis has been tested by Takahashi and colleagues, who used the exposure of pregnant rats with $\mathrm{sc}^{+/-}$genotype to carcinogen hydroquinone, as a designated second hit. The results of these experiments in young rats were disappointing with no histological alterations found in the $t \mathrm{sc}^{+/-}$genotype when compared to their $\mathrm{ssc}^{+/+}$ siblings [96]. In aged groups, however, where the advanced age was considered as the second hit, the authors report occasional histopathological changes, which resemble the human TSC pathology, including large dysmorphic pyramidal neurons in deep cortical layers (reactive for neuronal markers), abnormal cytomegalic cells (reactive for glial markers), and glial tumors. The presence of abnormally large neuron-like cells suggested that $t s c^{+/-}$rats might suffer from neuronal hyperexcitability which could contribute towards the generation of seizures. Strikingly, none of the Eker rats in this or subsequent studies showed signs of seizure.

Detailed analysis of the histochemistry and anatomical distribution of lesions in aged Eker rats has shown the prevalence of brain malformations in the $\mathrm{scc}^{+/-}$genotype from 30 to $60 \%$ of cases. Based on anatomical localization and histochemical characteristics, tubers have been divided into three subgroups $[73,82]$. Histopathological data show that the majority of lesions are directly associated with the ventricular system, with the remaining minority displaying also signs of their developmental origin from ependymal cells migrating into deeper brain compartments [72-74]. Although TSC distribution in the brain of $t s c^{+/-}$Eker rats is in general agreement with human data, there are some interesting specifics [9, 40, 97]. Unlike humans, in rats, subependymal malformations represent the most common lesion types. Like in humans, however, these nodular outgrowths in rats tend to develop, expand, and sometimes protrude into ventricles [9]. Subependymal hamartomas are especially prevalent in the region of the lateral ventricles and basal ganglia structures, and particularly within the caudate nucleus area and deep periventricular white matter $[9,10]$. Lesions have been frequently documented also in caudoputamen and nucleus accumbens area [95]. In a few cases, subependymal hamartia has been found also bilaterally, albeit these lesions had typically smaller size $(<2 \mathrm{~mm})[9,10]$. Similar to human TSC, lesions in the rat brain contained unusually large cells with abundant pale cytoplasm and multiple large nuclei [95]. Inside lesions, numerous elongated and highly fibrillated cell processes, referred to as Rosenthal fibers, are visible [9]. The latter appears to be enriched with astrocytic protein aggregates, prevalent during intense gliosis, including in Alexander disease and occasionally also in other tumors of glial origin [98].

In addition to subependymal hamartia associated with the ventricular system of the brain, lesions have also been found at a considerable distance from the ventricles, with most cases reported in the white matter $[9,10,95]$. In Eker rats, malformations have also been found at corticomedullary junctions of the frontoparietal convexity, in the corpus callosum [9, 10, 97], and the motor area [95]. Unlike large subependymal neoplasms, lesions in deep compartments are usually of smaller size and are less common ( $\sim 21 \%$ of examined rat brains) [9]. Kútna and colleagues recently reported a strongly calcified lesion within the piriform lobe of the aged Eker rat [10]. Figure 3(a1-5) presents typical examples of lesions distributed in various forebrain structures. The abnormally large cells ( $20-30 \mu \mathrm{m}$ in diameter) in rat lesions, like in humans, have characteristic cytoplasm, round or ovoid eccentric nucleus, with nucleoplasm containing 

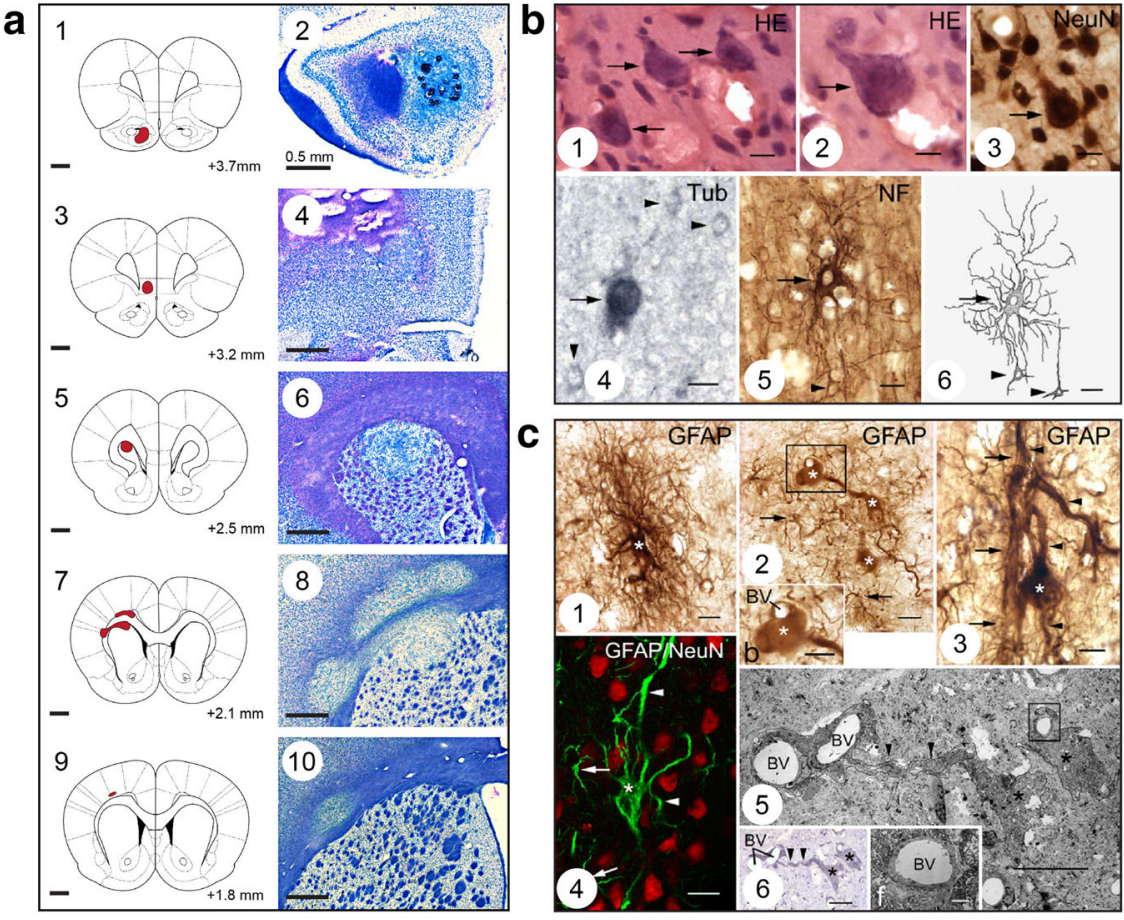

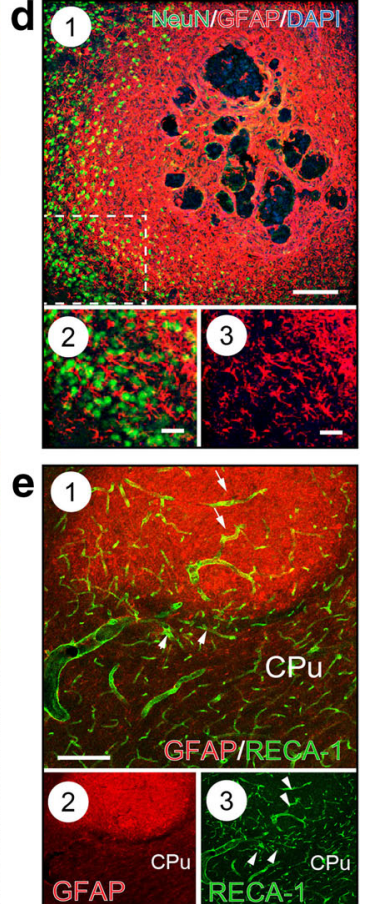

one cell (asterisk) surrounding a blood vessel (BV; inset c2). GFAP (glial fibrillary acidic protein)-positive astrocyte (asterisk) forms irregularly shaped and extended processes (arrowheads) associated with a blood vessel (arrows) (c3). Representative micrograph of a giant astrocyte immunopositive for GFAP in the cell body (asterisk) and processes (arrowheads), but immunonegative for NeuN (c4). Note NeuN staining (red) and GFAP (green) in normal neurons and astrocytes (arrows), respectively. Giant astrocytes (asterisks) and blood vessels (BV) in a semithin section (c5-6) and electron micrograph (c6). One astrocyte forms a giant end-foot (arrowheads). The other (box at right) surrounds a blood vessel. The boxed area with GFAP-positive astrocyte in higher magnification. Note the astrocyte surrounds a BV. Images were taken with permission from Wenzel et al. [97]. (d1) NeuN- (green) and GFAP (red)-positive cells in a lesion within the piriform lobe. Boxed area (right lower) shown at higher magnification demonstrating robust GFAP immunoreactivity with NeuN (d2) and GFAP alone (d3). (e1) GFAP and RECA-1 double immunofluorescence of the lesion area: low magnification micrographs showing tumor-related changes in density and distribution of RECA-1-labeled vascular profiles within the lesion area and at the interface with surrounding parenchyma. (e2-3) Decomposed GFAP and RECA-1 immunofluorescence images. $\mathrm{CPu}=$ caudateputamen. Images adapted with permission from Kutna et al. [10]

As noted earlier, in humans, cortical hamartia is the most prevalent, which are thought to be responsible for an array of neurological and psychiatric symptoms and especially for developing epilepsy $[70,99,100]$. In the literature on Eker rat models however, there is only one case reporting a cortical tuber. Indeed, Mizuguchi and colleagues described so far the only case of a single lesion in the primary motor area of a 19month-old $t s c^{+/-}$rat $[76,95]$. The morphological features of this tuber resemble human cortical lesions, i.e., show dysplasia with dyslamination, and a visibly reduced number and density of neurons within the affected region, along with to nonirradiated rats [97]. 
distinctive signs of astrogliosis [95]. Most of the cells in the cortical tuber appeared to be cytomegalic neurons $(\sim 35 \mathrm{~mm}$ in size) with abundant cytoplasm, large nuclei, and single nucleolus. The cytomegalic neurons displayed strong immunoreactivity for neuronal markers [95]. The follow-up reports failed to find cortical tubers in $\mathrm{tsc}^{+/-}$rats, even in a very old age group (18-24 months), confirming the very low incidents of lesions in this location $[9,10,96]$. No cortical hamartia could be found also in adult Eker rats $\left(t s c 2^{+/-}\right)$exposed to irradiation in the early postnatal period $[97,101]$.

Overall, these reports demonstrate a reliable approximation of the major anatomical and histopathological characteristics of human brain TSC in Eker rats, with the main discrepancy being very rare occasions of cortical lesions in rats. Given the prevalence of cortical lesions in humans and their alleged role in causing neurological and psychiatric symptoms, the lower incidents of cortical hamartia in rats could explain important neurobehavioral differences from humans.

\section{Histochemical Profile of Brain Lesions in Eker Rats}

Several reports have presented a detailed account of histopathological changes in the brain of Eker rats [9, 10, 96, 97, 101]. Initial studies of young animals described very minor alterations, which became more pronounced in older age $[9,11$, $76,95]$. The exposure of rats to irradiation and carcinogens (i.e., second hit) at younger age exacerbated histopathological changes at later time points, with cytomegalic neurons and giant astrocyte-like cells observed in various brain regions [97]. Analysis of cellular aberrations in the rat brain and comparison with those in humans has confirmed their close similarities, with reports of cytochemical profiles of lesions at different brain locations revealing multiple shared features $[9,97]$. A recent report presented a detailed characterization of lesion-related neuronal alterations, as well as glial changes and vascular remodeling [10], not described so far in human TSC. Figure $3 \mathrm{~b}$ to $\mathrm{d}$ summarizes the histopathological and cytochemical changes associated with brain malformations in Eker rat.

Takahashi and colleagues showed two types of atypical cells in brain lesions of aged Ekers: 1) swollen pyramidal neurons immune-reactive for NeuN, tuberin, and neuronal glutamate transporter EAAC-1, largely confined to the IVVI cortical layers, and 2) giant nondifferentiated cells, known also as cytomegalic cells with astrocytic appearance, labeled positive for GFAP, vimentin, and nestin [96]. Another report in adult Eker rats irradiated during the early postnatal period showed that in addition to giant astrocyte-like cells present in cortical or subcortical structures, there is a considerable increase in the number of dysmorphic large neurons $(>50 \mu \mathrm{m})$ in the cortex, as compared to nonirradiated rats [97]. Notably, the majority of atypical neuron-like cells maintain generic features of neurons, i.e., immunoreactivity for NeuN, neurofilament (NF), and MAP2. Interestingly, some atypical neurons showed also immunoreactivity for glial proteins (e.g., GFAP), as well as intermediate filament protein nestin [97]. Results of the analysis of a rare case of cortical hamartia in Eker rats demonstrated that the majority of cytomegalic neurons have abundant cytoplasm, large nuclei, and single nucleolus. The cytomegalic neurons in this cortical tuber also showed immunoreactivity for some characteristic neuronal markers [95], but were negative for others, such as neurofilament H (SMI-32) and NSE, with their axons displaying positivity for phosphorylated antineurofilament $\mathrm{H}$ protein (SMI31) $[76,95]$. Virtually none of the cortical neurons showed immunoreactivity for GFAP, calbindin, vimentin, or tuberin. Finally, a relatively small number of cells within lesions displayed immune-positivity for NSE, a marker for injured neurons [95].

Kutna and colleagues reported an extensive loss of neurons and axons associated with subcortical lesions, along with a strong presence of astrocytes and microglia in the core of hamartia [10]. Using specific markers for different neuronal subtypes, loss of tyrosine hydroxylase ( $\mathrm{TH}$ )-positive fibers, as well as degeneration of glutamatergic, and parvalbuminpositive GABAergic neurons and their axons were shown [10]. Nonetheless, some axons traversing TSC lesions remained intact, in agreement with reports showing occasional SMI-31- and $\beta$-III tubulin-positive (a marker of neurons) neurites and myelin basic protein-positive axons [9]. In irradiated Eker rats, strong immunoreactivity of lesions for GAD67 and MAP2 was also reported [97]. Despite the extensive neuronal loss and complex histopathological alterations, the principal and distinguishing feature of malformations in aged rats is the prevalence of glial pathology, with the occurrence of large reactive astrocytes and high expression of GFAP [9, 10, 97]. We have recently shown that a strong increase in the expression of the GFAP within TSC lesion and reduction of NeuNpositive cells is associated with an upsurge in overall cell number (i.e., DAPI-positive nuclei), attributable to the proliferation of nonneuronal cells [10]. Earlier, an increase in the expression of glutamine synthetase (GS), an astrocytic enzyme responsible for catalyzing the conversion of glutamate and ammonia to glutamine, has been also reported [102]. Using Iba1-specific antibody labeling microglia and circulating macrophages [103] with semiquantitative scoring of Iba1, NeuN, and DAPI expression, an increase in Ibal levels in lesions was also observed [10]. Compared to resting microglia, most of the Iba1positive cells display phagocytic features, with retracted processes and "amoeboid" morphology [10].

It is important to note also the high level of expression of progenitor cell markers, i.e., intermediate filaments nestin and 
vimentin, in hamartia of Eker rat [10]. Earlier studies showed evidence for enhanced nestin immunoreactivity in the brain of irradiated Eker rats [97], suggesting the presence of both immature neurons and astrocytes. These findings contrast with the results of Mizuguchi et al. [95], which did not observe vimentin immunoreactivity, with reasons for such inconsistency remaining unclear. Last but not the least, lesions in $t s c 2^{+/-}$rats show signs of vascular remodeling and angiogenesis, with the formation of a dense network of microvessels warranting nutrition and oxygen supply to the growing tissue [10]. Larger vessels in the core and higher density of capillaries at the interface of the lesion with healthy tissue, as evident from staining with RECA-1 endothelial cell marker, are in agreement with tuber-related vascular remodeling [10]. Overall, described alterations associated with malformations in the brain of the Eker rat replicate closely histopathological changes related to the human TSC and display multiple generic features of other nonmalignant tumors.

\section{Neurophysiological and Behavioral Phenotypes in the Eker Rat Model}

The majority of human brain pathologies with focal lesions and dysmorphic neurons and glia are linked to a range of neurological and psychiatric symptoms that affect various aspects of brain functions and behavior. As noted, epilepsy is the most common sign of human TSC, with mental retardation and dementia, autism, and other neurobehavioral impairments also observed at advanced stages of the disease [104, 105]. In animal models with disruptions in mTORC1 signaling, structural and functional modifications in the brain are viewed primarily in conjunction with the impairments of neuroplasticity and signaling mechanisms, which might affect cellular growth, proliferation, and differentiation, leading to mental retardation and cognitive deficit [106, 107].

The appearance of a considerable number of atypically large cells and focal lesions throughout the brain of adult nonirradiated and irradiated aged $t s c 2^{+/-}$rats are expected to lead to an array of neurobehavioral changes. Over more than two decades of research, however, numerous attempts have failed to detect neurophysiological and behavioral alterations in Eker rats. This contrasts with major electrophysiological and behavioral abnormalities in TSC humans, with epilepsy being the most widely observed symptom, reported in the majority of patients, and often appearing before 2 years of age [71]. Henceforth, despite that Eker rats recapitulate the molecular and cellular neuropathology of human TSC [96, 97], they do not generate spontaneous seizures, which is the case in humans [11, 108]. Experiments, where seizures have been observed in Eker rats, were typically induced by the "second hit" [11, 97]. Waltereit et al. reported that repeated intraperitoneal administration of $30 \mathrm{mg} / \mathrm{kg}$ pentylenetetrazole
(PTZ), a chemical second hit, induced seizures in 8-month-old Eker rats at an accelerated rate, as compared to wild-type control rats [11]. It was shown also that postnatal irradiation applied as a second hit lowered the latency to seizure induction after administering flurothyl [97], with irradiated rats displaying no spontaneous seizures [101]. In multiple $t s c 2^{+/-}$ rat studies, aging has been used as a second hit. Despite a significantly higher number of dysmorphic neurons in 17month-old Eker rats, no spontaneous seizures could be observed $[11,96]$. The extremely low indecent of cortical hamartia, including in rats that received the "second hit," has been considered as an explanation of the absence of epileptic seizures, a notion that needs experimental confirmation. Studies examining changes in microcircuit dynamics and alterations in neurophysiological properties of neurons in proximity to lesions ex vivo could elucidate the potential mechanistic link between TSC malformations and the onset of seizures, as demonstrated for epileptic activity related to amyloid plaques of Alzheimer's disease [109-111]. Investigations of hippocampal plasticity in 30- to 50-day-old Eker rats revealed elevated paired-pulse facilitation in the Schaffer collateralCA1 synapse of the hippocampus [112]. In the same preparation, long-term potentiation (LTP) and depression (LTD) two synaptic mechanisms that play a key role in regulating the neural network excitability and synaptic homeostasis, were found to be reduced. These findings imply subtle pathophysiological changes in Eker rats, which impair synaptic functions and plasticity but do not lead to the generation of seizures. Of note, in TSC patients, epileptiform discharges can occur in the absence of observable tubers $[113,114]$, indicating that more subtle molecular and functional alterations related to the hyperactivity of mTORC1 might be the primary cause of the epileptic activity. While further research of the Eker rat model is likely to continue to yield important mechanistic insights, other murine models also warrant attention and should facilitate elucidation of numerous complex aspects of TSC (Box 1).

Using neurobehavioral tests, several groups attempted to elucidate the memory impairments in young Eker rats $[9,11$, $95,96,121]$. In these studies, the only difference from controls was found in tasks challenging episodic-like memory, discovered in a delayed matching-to-place task [121]. All other behavioral tests assessing exploration and anxiety (light/dark box) as well as learning and memory (Pavlovian conditioned taste aversion test, Morris water maze with hidden platform task including reversal learning, and probe trials) did not reveal any behavioral constraints [11]. Subsequently, the same group reported epileptic condition following systemic administration of kainic acid, but with no differences in learning abilities (fear conditioning, fear extinction, and Morris water maze) [122]. In contrast, in the open field paradigm, novel object recognition and social exploration were significantly reduced in naïve rats and even more so in the kainate-treated 
Box 1 TSC mouse models with an overview of neuropathological and neurobehavioral characteristics

\section{TSC1 mouse models}

$T s c 1$ knockout mice: $t s c 1^{-/}$homozygous are not viable and die around embryonic days 10.5-11.5 due to neural tube defects at the head region. Heterozygote $t s c 1^{+/-}$mice develop renal tumors and hepatic hemangiomas, with loss of tuberin observed in situ inside lesions. $t s c 1^{+/-}$mice develop macroscopically visible renal carcinomas or renal cystadenomas by the age of 10 months. Overall, observed phenotypes of $t s \mathrm{I}^{+/-}$mice resembled those of $t s 2^{+/-}$knockout mice reported, in agreement with a shared pathway of tsc1 and tsc2 products. Of note, the development of renal tumors in $t s c 1^{+/-}$mice was slower than that in $t s c 2^{+/-}$mice. Brain lesions were not examined in this study [115].

$T s c 1^{+/-}$knockout mice: correlating cerebral lesions, epilepsy, and cognitive function with high-resolution MRI, immunohistochemistry, and behavioral tests such as fear conditioning, social interaction, nest building, and social interactions. No spontaneous seizures could be observed. No cerebral lesions or signs of cerebral pathology could be detected. Ultrastructural analysis revealed no differences in the spine density and dendritic branching of hippocampal granule cells. Tsc1 ${ }^{+/}$mice showed impaired learning in the hippocampus-sensitive learning tasks, and impaired social behavior, supporting the notion that haploinsufficiency for the TSC genes leads to aberrations in neuronal functioning resulting in impaired learning and social behavior [108].

$\mathrm{Tsc}^{\mathrm{C}^{-}} \mathrm{Syn} / \mathrm{Cre}^{+}$mice: $t s c 1$ "floxed" allele combined with a synapsin I promoter-driven Cre allele to achieve tsc1 expression in cortical neurons during embryonic development. Mutant mice display several neurological abnormalities beginning from early postnatal life, including spontaneous and stimulus-induced seizures, with some seizures ending in a fatal tonic phase before the age of 65 days. Many cortical and hippocampal cells are enlarged and dysplastic, enriched with phospho-S6. Axonal bundles show a striking delay in myelination. At P21, mice show laminar disorganization in the cortical region and the presence of large cells in the cortex and hippocampus. The majority of enlarged cells showed increase in pS6 immunoreactivity. Reduced myelination (MBP expression) is evident throughout the brain, but oligodendrocyte distribution was not changed [60].

Tsc1 ${ }^{\text {GFAP1 }}$ CKO mice: this mouse model has inactivated $t s c 1$ gene in glia ( $\mathrm{Tsc} 1{ }^{\mathrm{GFAP}} \mathrm{CKO}$ mice). The strain shows a shortened lifespan, which begins to die around 12 weeks, and shows pronounced changes in EEG reminiscent of seizures, with their onset after the first month of age. Histopathological and immunofluorescence analysis showed an increase in the number of GFAP immune-positive astrocytes in the brains of mutant mice. However, no lesions or tumors of glial origin were noted in these mice. The model also shows an increase in the number of proliferating PCNA ${ }^{+}$cells [116] as well as expression of P-S6 (Zeng et al. [117]), compared with controls. Subsequent examination of adult Tsc ${ }^{\text {GFAP }}$ mice revealed that cortical and hippocampal astrocytes express vimentin and brain lipid-binding protein (BLBP) [92].

TSC2 mouse models

Tsc2 loss of function: homozygous $t s c^{2-/}$ mice like $t s c^{2--}$ Eker rats are not viable and die during embryonal development days 9.5-12.5 because of liver hypoplasia, indicating that tuberin plays an essential role in development. $t s \mathrm{~S}^{+/-}$adult mice develop a $100 \%$ incidence of multiple bilateral renal cystadenomas, 50\% incidence of liver hemangiomas, and 32\% incidence of lung adenomas by 15 months of age. Progression to renal carcinomas, fatal bleeding from the liver hemangiomas, and angiosarcomas all occur at a rate of less than $10 \%$. The renal cystadenomas develop from intercalated cells of the cortical collecting duct and uniformly express gelsolin at high levels, which enables the detection of early lesions. The tumor distribution is influenced by the genetic background of the primary mouse line. Tumors occur in the kidneys, liver, and lungs. This study does not report cerebral lesions in the $t s c 2^{+/-}$mice [118].

Tsc $2^{\text {flox/ko }}$,hGFAP-Cre mice: this mouse model has a selective and inducible deletion of the $t s c 2$ gene in radial glial progenitor cells of the developing cerebral cortex and hippocampus. All progenitor cells derived from neurons and astrocytes of $t s c 2^{-/}$radial glia show significant loss of tuberin antigen. Animals appear runted, and were on average smaller, and died around 4 weeks; therefore, all analyses were made at P21. Increased levels of phosphorylated (Ser 240/244) S6 (pS6) indicate activation of the mTORC1 pathway. At this age, mice also have a thicker cortex and apparent defects in cortical lamination and hippocampal organization, reminiscent of those observed in Eker rats. In these mice, GFAP expression is significantly increased in the entire cortex, in the hippocampus, and around lateral ventricles [119].

Tsc2 ${ }^{\mathrm{GFAP} 1} \mathrm{CKO}$ mice: this model is characterized by the inactivation of $t s c 2$ gene in astrocytes (Tsc1 ${ }^{\mathrm{GFAP}} \mathrm{CKO}$ mice). Tsc $2{ }^{\mathrm{GFAP} 1} \mathrm{CKO}$ mice have $100 \%$ mortality by 10 weeks and have an earlier onset and higher frequency of seizures in comparison with Tsc $1{ }^{\mathrm{GFAP1}} \mathrm{CKO}$ mice. Histochemical analysis of the brain demonstrates severe abnormalities, with the number of astrocytes considerably increased, while the expression of the astrocyte glutamate transporter GLT-1 reduced, as compared with controls. Histological analysis shows evidence for neuronal disorganization in the hippocampus. As expected, inactivation of $t s c 2$ exhibits increased activation of mTOR, with increased P-S6 expression in astrocytes. The increased astroglial proliferation, neuronal disorganization, and resulting megalencephaly are more severe in $\mathrm{Tsc} 2{ }^{\mathrm{GFAP} 1} \mathrm{CKO}$ mice compared with Tsc $1{ }^{\text {GFAP1 }}$ CKO mice [117].

NEX-Tsc2 mouse line: in this mouse model, $t s c 2$ gene is deleted specifically in postmitotic excitatory neurons of the embryonic forebrain, with $t s c 2$ in progenitor cells, glia, or inhibitory neurons remaining nonaffected. The homozygous NEX-tsc2 mice die prematurely at around 16 postnatal days, while the lifespan of heterozygote mice remaining unaffected. Neuronal abnormalities of heterozygote NEX-tsc2 mice included the presence of 
Box 1 (continued)

an abnormally thick cellular layer in the deep neocortex and dispersed pyramidal cells in hippocampal area CA1. No signs of astrogliosis in the cortex of NEX-tsc2 were noted. The mouse line has not to be scored for neurobehavioral or neurophysiological changes [120].

group. The authors concluded that $t s c 2$ haploinsufficiency and epileptic seizures might additively compromise social interaction, a finding requiring independent verification.

Taken as a whole, despite faithfully replicating cellular and molecular pathology and some specific features of human brain lesions, the utility of $t s c 2^{+/-}$Eker rats as a model for neurological or behavioral symptoms remains controversial and requires further experimental analysis. Enhanced episodic-like memory with abnormal short-term plasticity and reduced theta-burst stimulation-induced LTP require cautious interpretation in the context of neuropathological changes and absence of overt behavioral phenotype.

\section{Summary and Conclusions}

One of the key priorities of translational neurobiology is elucidating functional and molecular processes in the brain underlying neurological and psychiatric diseases, to enable effective medical interventions. In neurology and especially in psychiatry, the association between alleged genetic causes and clinical manifestations of the disease is often tentative and may vary to a considerable degree. TSC presents an archetypal example of widely variable neurological symptoms induced by TSC1/TSC2 loss of function, with underlying mechanisms for the phenotypic heterogeneity remaining a matter of debate. Indeed, while clinical reports of TSC in humans pinpoint refractory seizures as one of the main manifestations of the pathology, almost a third of TSC cases do not display signs of seizures. Such inconsistency implies complex underlying mechanisms and compensatory adjustments, which render the management of the condition a major therapeutic challenge. It also highlights the need for identifying reliable functional and molecular biomarkers of the disease, to enable early diagnosis with grading and characterization of cause-symptom (i.e., genotype-phenotype) relationship and timely intervention.

Loss-of-function mutations in $t s c 2$ in Eker rats, like in humans, are expected to result in an array of neurological and psychiatric symptoms. Indeed, the shared cause and molecular pathology render $t s c 2^{+/-}$a promising model for studies of TSC from neuropathological, neurophysiological, and behavioral perspectives. Despite many years of research with the use of this model and considerable progress, important questions remain concerning the extent of replication of the functional and neurodevelopmental human TSC phenotype in rats. While the $t s c 2^{+-}$rats recapitulate most of the features of cellular and molecular pathology, the vast majority of abnormalities in rats set on at advanced age, unlike in humans with histopathological alterations evident from a very young age. Major discrepancies are also found in terms of the anatomical distribution of lesions throughout the brain, with cortical TSC, while the most prevalent in humans, not been conclusively shown in Eker rats. Finally, important questions remain over the neurobehavioral manifestations of TSC pathology, with the majority of neurological and psychiatric symptoms prevalent in humans not detected in the Eker rat. At this stage, there are no ready explanations for major discrepancies in the disease onset, symptomology, and differential sensitivity of various brain regions to TCS1/TSC2 loss of function. In these regards, the role of a variety of genetic (i.e., mutational burden, penetrance, the expressivity of TSC genes) and environmental (i.e., the second hit) factors and comorbidities has been considered. Given that TSC deficit decouples mTORC1 from upstream signaling, an array of functional changes are expected which contribute towards complex mechanisms and neurobehavioral manifestations of the disease. Wide-ranging symptomatic deviations of human and rat TSCs, despite shared genetic cause and molecular pathology, testify incompleteness of the current understanding of this complex condition and urge for further research and cautious interpretation of the functional and neurobehavioral data.

Supplementary Information The online version contains supplementary material available at https://doi.org/10.1007/s13311-020-01000-7.

Acknowledgments This study was supported by the project Sustainability for the National Institute of Mental Health, under grant number LO1611, with financial support from the Ministry of Education, Youth and Sports of the Czech Republic under the NPU I program.

Required Author Forms Disclosure forms provided by the authors are available with the online version of this article.

\section{References}

1. Ryskalin, L., et al., mTOR-Dependent Cell Proliferation in the Brain. Biomed Res Int, 2017. 2017: p. 7082696.

2. Crino, P.B., mTOR signaling in epilepsy: insights from malformations of cortical development. Cold Spring Harb Perspect Med, 2015. 5(4):a022442. https://doi.org/10.1101/ cshperspect.a022442.

3. Saxton, R.A. and D.M. Sabatini, mTOR Signaling in Growth, Metabolism, and Disease. Cell, 2017. 169(2): p. 361-371.

4. Dentel, B., C.O. Escamilla, and P.T. Tsai, Therapeutic Targeting of mTORC2 in mTORopathies. Neuron, 2019. 104(6): p. 10321033. 
5. Richardson, E.P., Jr., Pathology of tuberous sclerosis. Neuropathologic aspects. Ann N Y Acad Sci, 1991. 615: p. 128-139.

6. Vinters, H.V., et al., Cortical dysplasia, genetic abnormalities and neurocutaneous syndromes. Dev Neurosci, 1999. 21(3-5): p. 248259.

7. Eker, R., Familial renal adenomas in Wistar rats; a preliminary report. Acta Pathol Microbiol Scand, 1954. 34(6): p. 554-562.

8. Eker, R., et al., Hereditary renal adenomas and adenocarcinomas in rats. Diagn Histopathol, 1981. 4(1): p. 99-110.

9. Yeung, R.S., C.D. Katsetos, and A. Klein-Szanto, Subependymal astrocytic hamartomas in the Eker rat model of tuberous sclerosis. Am J Pathol, 1997. 151(5): p. 1477-1486.

10. Kutna, V., et al., Tuberous Sclerosis (tsc2+/-) Model Eker Rats Reveals Extensive Neuronal Loss with Microglial Invasion and Vascular Remodeling Related to Brain Neoplasia. Neurotherapeutics, 2020. 17(1): p. 329-339.

11. Waltereit, R., et al., Enhanced episodic-like memory and kindling epilepsy in a rat model of tuberous sclerosis. J Neurochem, 2006. 96(2): p. 407-413.

12. Rennebeck, G., et al., Loss of function of the tuberous sclerosis 2 tumor suppressor gene results in embryonic lethality characterized by disrupted neuroepithelial growth and development. Proc Natl Acad Sci U S A, 1998. 95(26): p. 15629-15634.

13. Knudson, A.G., Jr., Mutation and cancer: statistical study of retinoblastoma. Proc Natl Acad Sci U S A, 1971. 68(4): p. 820-823.

14. Tomlinson, I.P., R. Roylance, and R.S. Houlston, Two hits revisited again. J Med Genet, 2001. 38(2): p. 81-85.

15. Afshar Saber, W. and M. Sahin, Recent advances in human stem cell-based modeling of Tuberous Sclerosis Complex. Mol Autism, 2020. 11(1): p. 16.

16. Blair, J.D. and H.S. Bateup, New frontiers in modeling tuberous sclerosis with human stem cell-derived neurons and brain organoids. Dev Dyn, 2020. 249(1): p. 46-55.

17. Robertson, F.L., et al., Experimental models and tools to tackle glioblastoma. Dis Model Mech, 2019. 12(9):dmm040386. https:// doi.org/10.1242/dmm.040386.

18. Parsa, A.T., et al., Limitations of the C6/Wistar rat intracerebral glioma model: implications for evaluating immunotherapy. Neurosurgery, 2000. 47(4): p. 993-999; discussion 999-1000.

19. Workman, P., et al., Guidelines for the welfare and use of animals in cancer research. Br J Cancer, 2010. 102(11): p. 1555-1577.

20. Simons, B.W. and C. Brayton, Challenges and Limitations of Mouse Xenograft Models of Cancer. Patient Derived Tumor Xenograft Models, ed. R. Uthamanthil and P. Tinkey. 2016, Johns Hopkins University School of Medicine, Baltimore, MD, United States: Academic Press.

21. Kim, K.M., et al., Failure of a patient-derived xenograft for brain tumor model prepared by implantation of tissue fragments. Cancer Cell Int, 2016. 16: p. 43.

22. Fryer, A.E., et al., Evidence that the gene for tuberous sclerosis is on chromosome 9. Lancet, 1987. 1(8534): p. 659-661.

23. van Slegtenhorst, M., et al., Identification of the tuberous sclerosis gene TSC1 on chromosome 9q34. Science, 1997. 277(5327): p. 805-808.

24. Kandt, R.S., et al., Linkage of an important gene locus for tuberous sclerosis to a chromosome 16 marker for polycystic kidney disease. Nat Genet, 1992. 2(1): p. 37-41.

25. Henske, E.P., et al., Tuberous sclerosis complex. Nat Rev Dis Primers, 2016. 2: p. 16035.

26. Lam, H.C., B.J. Siroky, and E.P. Henske, Renal disease in tuberous sclerosis complex: pathogenesis and therapy. Nat Rev Nephrol, 2018. 14(11): p. 704-716.

27. Bongaarts, A., et al., Subependymal giant cell astrocytomas in Tuberous Sclerosis Complex have consistent TSC1/TSC2 biallelic inactivation, and no BRAF mutations. Oncotarget, 2017. 8(56): p. 95516-95529.

28. Huang, J. and B.D. Manning, The TSC1-TSC2 complex: a molecular switchboard controlling cell growth. Biochem J, 2008. 412(2): p. 179-190.

29. Rosset, C., C.B.O. Netto, and P. Ashton-Prolla, TSC1 and TSC2 gene mutations and their implications for treatment in Tuberous Sclerosis Complex: a review. Genet Mol Biol, 2017. 40(1): p. 6979.

30. Lamb, R.F., et al., The TSC1 tumour suppressor hamartin regulates cell adhesion through ERM proteins and the GTPase Rho. Nat Cell Biol, 2000. 2(5): p. 281-287.

31. Haddad, L.A., et al., The TSC1 tumor suppressor hamartin interacts with neurofilament-L and possibly functions as a novel integrator of the neuronal cytoskeleton. J Biol Chem, 2002. 277(46): p. $44180-44186$.

32. Schopel, M., et al., The small GTPases Ras and Rheb studied by multidimensional NMR spectroscopy: structure and function. Biol Chem, 2017. 398(5-6): p. 577-588.

33. Fehon, R.G., A.I. McClatchey, and A. Bretscher, Organizing the cell cortex: the role of ERM proteins. Nat Rev Mol Cell Biol, 2010. 11(4): p. 276-287.

34. Kleijer, K.T., et al., Neurobiology of autism gene products: towards pathogenesis and drug targets. Psychopharmacology, 2014. 231(6): p. 1037-1062.

35. Kobayashi, T., et al., Identification of a leader exon and a core promoter for the rat tuberous sclerosis 2 (Tsc2) gene and structural comparison with the human homolog. Mamm Genome, 1997. 8(8): p. 554-558.

36. Maheshwar, M.M., et al., The GAP-related domain of tuberin, the product of the TSC2 gene, is a target for missense mutations in tuberous sclerosis. Hum Mol Genet, 1997. 6(11): p. 1991-1996.

37. Nussinov, R., et al., The Mystery of Rap1 Suppression of Oncogenic Ras. Trends Cancer, 2020. 6(5): p. 369-379.

38. Jaskiewicz, A., B. Pajak, and A. Orzechowski, The Many Faces of Rap1 GTPase. Int J Mol Sci, 2018. 19(10):2848. https://doi.org/ 10.3390/ijms19102848.

39. Rubinfeld, B., et al., Molecular cloning of a GTPase activating protein specific for the Krev-1 protein p21rap1. Cell, 1991. 65(6): p. 1033-1042.

40. Yeung, R.S., Lessons from the Eker rat model: from cage to bedside. Curr Mol Med, 2004. 4(8): p. 799-806.

41. Soucek, T., et al., Role of the tuberous sclerosis gene- 2 product in cell cycle control. Loss of the tuberous sclerosis gene-2 induces quiescent cells to enter S phase. J Biol Chem, 1997. 272(46): p. 29301-29308.

42. Jin, M., Q. An, and L. Wang, Importance of tuberin in carcinogenesis. Oncol Lett, 2017. 14(3): p. 2598-2602.

43. Rosner, M. and M. Hengstschlager, Cytoplasmic/nuclear localization of tuberin in different cell lines. Amino Acids, 2007. 33(4): p. 575-579.

44. Wienecke, R., et al., Co-localization of the TSC2 product tuberin with its target Rap1 in the Golgi apparatus. Oncogene, 1996. 13(5): p. 913-923.

45. Demetriades, C., N. Doumpas, and A.A. Teleman, Regulation of TORC1 in response to amino acid starvation via lysosomal recruitment of TSC2. Cell, 2014. 156(4): p. 786-799.

46. Demetriades, C., M. Plescher, and A.A. Teleman, Lysosomal recruitment of TSC2 is a universal response to cellular stress. Nat Commun, 2016. 7: p. 10662.

47. Johnson, M.W., et al., Hamartin and tuberin expression in human tissues. Mod Pathol, 2001. 14(3): p. 202-210.

48. $\mathrm{Li}, \mathrm{Y}$., et al., Regionally specific TSC1 and TSC2 gene expression in tuberous sclerosis complex. Sci Rep, 2018. 8(1): p. 13373. 
49. Dan, H.C., et al., Phosphatidylinositol 3-kinase/Akt pathway regulates tuberous sclerosis tumor suppressor complex by phosphorylation of tuberin. J Biol Chem, 2016. 291(43): p. 22848.

50. Inoki, K., et al., TSC2 is phosphorylated and inhibited by Akt and suppresses mTOR signalling. Nat Cell Biol, 2002. 4(9): p. 648657

51. Potter, C.J., L.G. Pedraza, and T. Xu, Akt regulates growth by directly phosphorylating Tsc2. Nat Cell Biol, 2002. 4(9): p. 658665 .

52. Tee, A.R., et al., Tuberous sclerosis complex-1 and -2 gene products function together to inhibit mammalian target of rapamycin (mTOR)-mediated downstream signaling. Proc Natl Acad Sci U S A, 2002. 99(21): p. 13571-13576.

53. Ma, L., et al., Identification of S664 TSC2 phosphorylation as a marker for extracellular signal-regulated kinase mediated mTOR activation in tuberous sclerosis and human cancer. Cancer Res, 2007. 67(15): p. 7106-7112.

54. Nie, D., et al., Tsc2-Rheb signaling regulates EphA-mediated axon guidance. Nat Neurosci, 2010. 13(2): p. 163-172.

55. Han, S., et al., Pam (Protein associated with Myc) functions as an E3 ubiquitin ligase and regulates TSC/mTOR signaling. Cell Signal, 2008. 20(6): p. 1084-1091.

56. Inoki, K., T. Zhu, and K.L. Guan, TSC2 mediates cellular energy response to control cell growth and survival. Cell, 2003. 115(5): p. 577-590.

57. Hahn-Windgassen, A., et al., Akt activates the mammalian target of rapamycin by regulating cellular ATP level and AMPK activity. J Biol Chem, 2005. 280(37): p. 32081-32089.

58. Lee, D.F., et al., IKK beta suppression of TSC1 links inflammation and tumor angiogenesis via the mTOR pathway. Cell, 2007. 130(3): p. 440-455.

59. Kwiatkowski, D.J., Rhebbing up mTOR: new insights on TSC1 and TSC2, and the pathogenesis of tuberous sclerosis. Cancer Biol Ther, 2003. 2(5): p. 471-476.

60. Meikle, L., et al., A mouse model of tuberous sclerosis: neuronal loss of Tsc1 causes dysplastic and ectopic neurons, reduced myelination, seizure activity, and limited survival. J Neurosci, 2007. 27(21): p. 5546-5558.

61. Goncharova, E.A., et al., Tuberin regulates p70 S6 kinase activation and ribosomal protein S6 phosphorylation. A role for the TSC 2 tumor suppressor gene in pulmonary lymphangioleiomyomatosis (LAM). J Biol Chem, 2002. 277(34): p. 30958-30967.

62. Cheadle, J.P., et al., Molecular genetic advances in tuberous sclerosis. Hum Genet, 2000. 107(2): p. 97-114.

63. Napolioni, V. and P. Curatolo, Genetics and molecular biology of tuberous sclerosis complex. Curr Genomics, 2008. 9(7): p. 475487.

64. Osborne, J.P., A. Fryer, and D. Webb, Epidemiology of tuberous sclerosis. Ann N Y Acad Sci, 1991. 615: p. 125-127.

65. Samueli, S., et al., Tuberous Sclerosis Complex: new criteria for diagnostic work-up and management. Wien Klin Wochenschr, 2015. 127(15-16): p. 619-630.

66. Prather, P. and P.J. de Vries, Behavioral and cognitive aspects of tuberous sclerosis complex. J Child Neurol, 2004. 19(9): p. 666674.

67. de Vries, P.J., et al., A clinical update on tuberous sclerosis complex-associated neuropsychiatric disorders (TAND). Am J Med Genet C: Semin Med Genet, 2018. 178(3): p. 309-320.

68. Gipson, T.T., et al., Potential for treatment of severe autism in tuberous sclerosis complex. World J Clin Pediatr, 2013. 2(3): p. $16-25$.

69. Hunt, A. and J. Dennis, Psychiatric disorder among children with tuberous sclerosis. . Dev Med Child Neurol , 1987. 29: p. 190198.
70. Holmes, G.L., C.E. Stafstrom, and G. Tuberous Sclerosis Study, Tuberous sclerosis complex and epilepsy: recent developments and future challenges. Epilepsia, 2007. 48(4): p. 617-630.

71. Nabbout, R., et al., Epilepsy in tuberous sclerosis complex: Findings from the TOSCA Study. Epilepsia Open, 2019. 4(1): p. 73-84.

72. Braffman, B.H., et al., MR imaging of tuberous sclerosis: pathogenesis of this phakomatosis, use of gadopentetate dimeglumine, and literature review. Radiology, 1992. 183(1): p. 227-238.

73. Ridler, K., et al., Standardized whole brain mapping of tubers and subependymal nodules in tuberous sclerosis complex. J Child Neurol, 2004. 19(9): p. 658-665.

74. Peters, J.M., et al., Diffusion tensor imaging and related techniques in tuberous sclerosis complex: review and future directions. Future Neurol, 2013. 8(5): p. 583-597.

75. Yamanouchi, H., et al., Giant cells in cortical tubers in tuberous sclerosis showing synaptophysin-immunoreactive halos. Brain and Development, 1997. 19(1): p. 21-24.

76. Mizuguchi, M. and S. Takashima, Neuropathology of tuberous sclerosis. Brain and Development, 2001. 23(7): p. 508-515.

77. Grajkowska, W., et al., Brain lesions in tuberous sclerosis complex. ReviewFolia Neuropathol, 2010. 48(3): p. 139-149.

78. Fohlen, M., et al., Refractory epilepsy in preschool children with tuberous sclerosis complex: Early surgical treatment and outcome. Seizure, 2018. 60: p. 71-79.

79. Kalantari, B.N. and N. Salamon, Neuroimaging of tuberous sclerosis: spectrum of pathologic findings and frontiers in imaging. AJR Am J Roentgenol, 2008. 190(5): p. W304-W309.

80. Yamanouchi, H., et al., Evidence of abnormal differentiation in giant cells of tuberous sclerosis. Pediatr Neurol, 1997. 17(1): p. 49-53.

81. Henske, E.P., et al., Loss of tuberin in both subependymal giant cell astrocytomas and angiomyolipomas supports a two-hit model for the pathogenesis of tuberous sclerosis tumors. Am J Pathol, 1997. 151(6): p. 1639-1647.

82. DiMario, F.J., Jr., Brain abnormalities in tuberous sclerosis complex. J Child Neurol, 2004. 19(9): p. 650-657.

83. Goh, S., W. Butler, and E.A. Thiele, Subependymal giant cell tumors in tuberous sclerosis complex. Neurology, 2004. 63(8): p. $1457-1461$.

84. Adriaensen, M.E., et al., Prevalence of subependymal giant cell tumors in patients with tuberous sclerosis and a review of the literature. Eur J Neurol, 2009. 16(6): p. 691-696.

85. Cuccia, V., et al., Subependymal giant cell astrocytoma in children with tuberous sclerosis. Childs Nerv Syst, 2003. 19(4): p. 232243.

86. Buccoliero, A.M., et al., Subependymal giant cell astrocytoma (SEGA): Is it an astrocytoma? Morphological, immunohistochemical and ultrastructural study. Neuropathology, 2009. 29(1): p. 2530 .

87. Hirose, T., et al., Tuber and subependymal giant cell astrocytoma associated with tuberous sclerosis: an immunohistochemical, ultrastructural, and immunoelectron and microscopic study. Acta Neuropathol, 1995. 90(4): p. 387-399.

88. Di Rocco, C., A. Iannelli, and E. Marchese, On the treatment of subependymal giant cell astrocytomas and associated hydrocephalus in tuberous sclerosis. Pediatr Neurosurg, 1995. 23(3): p. 115121.

89. Crino, P.B., K.L. Nathanson, and E.P. Henske, The tuberous sclerosis complex. N Engl J Med, 2006. 355(13): p. 1345-1356.

90. Kim, J.Y., et al., Subependymal Giant Cell Astrocytoma Presenting with Tumoral Bleeding: A Case Report. Brain Tumor Res Treat, 2017. 5(1): p. 37-41.

91. Ess, K.C., et al., Developmental origin of subependymal giant cell astrocytoma in tuberous sclerosis complex. Neurology, 2005. 64(8): p. 1446-1449. 
92. Ess, K.C., et al., Expression profiling in tuberous sclerosis complex (TSC) knockout mouse astrocytes to characterize human TSC brain pathology. Glia, 2004. 46(1): p. 28-40.

93. Feng, L., M.E. Hatten, and N. Heintz, Brain lipid-binding protein (BLBP): a novel signaling system in the developing mammalian CNS. Neuron, 1994. 12(4): p. 895-908.

94. Jozwiak, S., et al., Tuberin and hamartin expression is reduced in the majority of subependymal giant cell astrocytomas in tuberous sclerosis complex consistent with a two-hit model of pathogenesis. J Child Neurol, 2004. 19(2): p. 102-106.

95. Mizuguchi, M., et al., Novel cerebral lesions in the Eker rat model of tuberous sclerosis: cortical tuber and anaplastic ganglioglioma. J Neuropathol Exp Neurol, 2000. 59(3): p. 188-196.

96. Takahashi, D.K., et al., Abnormal cortical cells and astrocytomas in the Eker rat model of tuberous sclerosis complex. Epilepsia, 2004. 45(12): p. 1525-1530.

97. Wenzel, H.J., et al., Morphology of cerebral lesions in the Eker rat model of tuberous sclerosis. Acta Neuropathol, 2004. 108(2): p. 97-108.

98. Wippold, F.J., 2nd, A. Perry, and J. Lennerz, Neuropathology for the neuroradiologist: Rosenthal fibers. AJNR Am J Neuroradiol, 2006. 27(5): p. 958-961.

99. Curatolo, P., et al., Neuropsychiatric aspects of tuberous sclerosis. Ann N Y Acad Sci, 1991. 615: p. 8-16.

100. Almobarak, S., et al., Tuberous Sclerosis Complex: Clinical Spectrum and Epilepsy: A Retrospective Chart Review Study. Transl Neurosci, 2018. 9: p. 154-160.

101. Tschuluun, N., H.J. Wenzel, and P.A. Schwartzkroin, Irradiation exacerbates cortical cytopathology in the Eker rat model of tuberous sclerosis complex, but does not induce hyperexcitability. Epilepsy Res, 2007. 73(1): p. 53-64.

102. Cooper, A.J., The role of glutamine synthetase and glutamate dehydrogenase in cerebral ammonia homeostasis. Neurochem Res, 2012. 37(11): p. 2439-2355.

103. Ito, D., et al., Enhanced expression of Iba1, ionized calciumbinding adapter molecule 1 , after transient focal cerebral ischemia in rat brain. Stroke, 2001. 32(5): p. 1208-1215.

104. Kirschstein, T., Synaptic plasticity and learning in animal models of tuberous sclerosis complex. Neural Plast, 2012. 2012: p. 279834.

105. Lee, B.H., T. Smith, and A.R. Paciorkowski, Autism spectrum disorder and epilepsy: Disorders with a shared biology. Epilepsy Behav, 2015. 47: p. 191-201.

106. Switon, K., et al., Tuberous sclerosis complex: From molecular biology to novel therapeutic approaches. IUBMB Life, 2016. 68(12): p. 955-962.

107. Switon, K., et al., Molecular neurobiology of mTOR. Neuroscience, 2017. 341: p. 112-153.

108. Goorden, S.M., et al., Cognitive deficits in Tsc1+/- mice in the absence of cerebral lesions and seizures. Ann Neurol, 2007. 62(6): p. 648-655.
109. Ovsepian, S.V., et al., Ambient Glutamate Promotes Paroxysmal Hyperactivity in Cortical Pyramidal Neurons at Amyloid Plaques via Presynaptic mGluR1 Receptors. Cereb Cortex, 2017. 27(10): p. 4733-4749.

110. Ovsepian, S.V. and V.B. O'Leary, Neuronal activity and amyloid plaque pathology: an update. J Alzheimers Dis, 2016. 49(1): p. 1319.

111. Ovsepian, S.V., et al., Amyloid Plaques of Alzheimer's Disease as Hotspots of Glutamatergic Activity. Neuroscientist, 2019. 25(4): p. 288-297.

112. von der Brelie, C., et al., Impaired synaptic plasticity in a rat model of tuberous sclerosis. Eur J Neurosci, 2006. 23(3): p. 686-692.

113. Chu-Shore, C., et al., The natural history of epilepsy in tuberous sclerosis complex. , ed. Epilepsia. 2010, John Wiley \& Sons, Ltd; 2010;51(7):1236-1241.

114. Vignoli, A., et al., Epilepsy in TSC: Certain etiology does not mean certain prognosis. Epilepsia. . 2013: John Wiley \& Sons, Ltd.

115. Kobayashi, T., et al., A germ-line Tsc1 mutation causes tumor development and embryonic lethality that are similar, but not identical to, those caused by Tsc2 mutation in mice. Proc Natl Acad Sci U S A, 2001. 98(15): p. 8762-8767.

116. Uhlmann, E.J., et al., Astrocyte-specific TSC1 conditional knockout mice exhibit abnormal neuronal organization and seizures. Ann Neurol, 2002. 52(3): p. 285-296.

117. Zeng, L.H., et al., Tsc2 gene inactivation causes a more severe epilepsy phenotype than Tsc1 inactivation in a mouse model of tuberous sclerosis complex. Hum Mol Genet, 2011. 20(3): p. 445454.

118. Onda, H., et al., Tsc2(+/-) mice develop tumors in multiple sites that express gelsolin and are influenced by genetic background. J Clin Invest, 1999. 104(6): p. 687-695.

119. Way, S.W., et al., Loss of Tsc2 in radial glia models the brain pathology of tuberous sclerosis complex in the mouse. Hum Mol Genet, 2009. 18(7): p. 1252-1265.

120. Crowell, B., et al., Complex Neurological Phenotype in Mutant Mice Lacking Tsc2 in Excitatory Neurons of the Developing Forebrain(123). eNeuro, 2015. 2(6):ENEURO.0046-15.2015. https://doi.org/10.1523/ENEURO.0046-15.2015.

121. Steele, R.J. and R.G. Morris, Delay-dependent impairment of a matching-to-place task with chronic and intrahippocampal infusion of the NMDA-antagonist D-AP5. Hippocampus, 1999. 9(2): p. 118-136

122. Waltereit, R., et al., Epilepsy and Tsc2 haploinsufficiency lead to autistic-like social deficit behaviors in rats. Behav Genet, 2011. 41(3): p. 364-372.

Publisher's Note Springer Nature remains neutral with regard to jurisdictional claims in published maps and institutional affiliations. 